\title{
Corticosteroid-Dependent Plasticity Mediates Compulsive Alcohol Drinking in Rats
}

\author{
Leandro F. Vendruscolo, ${ }^{1}$ Estelle Barbier, ${ }^{3}$ Joel E. Schlosburg, ${ }^{1}$ Kaushik K. Misra, ${ }^{1}$ Timothy W. Whitfield Jr, ${ }^{1}$ \\ Marian L. Logrip, ${ }^{1}$ Catherine Rivier, ${ }^{4}$ Vez Repunte-Canonigo, ${ }^{2}$ Eric P. Zorrilla, ${ }^{1}$ Pietro P. Sanna, ${ }^{2}$ Markus Heilig, ${ }^{3}$ \\ and George F. Koob ${ }^{1}$ \\ ${ }^{1}$ Committee on the Neurobiology of Addictive Disorders and ${ }^{2}$ Molecular and Integrative Neuroscience Department, The Scripps Research Institute, La Jolla, \\ California 92037, ${ }^{3}$ Laboratory of Clinical and Translational Studies, National Institute on Alcohol Abuse and Alcoholism, Bethesda, Maryland 20892-1108, \\ and ${ }^{4}$ The Clayton Foundation Laboratories for Peptide Biology, The Salk Institute, La Jolla, California 92037
}

Alcoholism is characterized by a compulsion to seek and ingest alcohol, loss of control over intake, and the emergence of a negative emotional state during abstinence. We hypothesized that sustained activation of neuroendocrine stress systems (e.g., corticosteroid release via the hypothalamic-pituitary-adrenal axis) by alcohol intoxication and withdrawal and consequent alterations in glucocorticoid receptor (GR) and mineralocorticoid receptor (MR) activation drive compulsive alcohol drinking. Our results showed that rats exposed to alcohol vapor to the point of dependence displayed increased alcohol intake, compulsive drinking measured by progressive-ratio responding, and persistent alcohol consumption despite punishment, assessed by adding quinine to the alcohol solution, compared with control rats that were not exposed to alcohol vapor. No group differences were observed in the self-administration of saccharinsweetened water. Acute alcohol withdrawal was accompanied by downregulated GR mRNA in various stress/reward-related brain regions [i.e., prefrontal cortex, nucleus accumbens (NAc), and bed nucleus of the stria terminalis (BNST)], whereas protracted alcohol abstinence was accompanied by upregulated GR mRNA in the NAc core, ventral BNST, and central nucleus of the amygdala. No significant alterations in MR mRNA levels were found. Chronic GR antagonism with mifepristone (RU38486) prevented the escalation of alcohol intake and compulsive responding induced by chronic, intermittent alcohol vapor exposure. Chronic treatment with mifepristone also blocked escalated alcohol drinking and compulsive responding during protracted abstinence. Thus, the GR system appears to be involved in the development of alcohol dependence and may represent a potential pharmacological target for the treatment of alcoholism.

\section{Introduction}

Alcoholism is a complex psychiatric condition characterized by compulsive alcohol seeking and ingestion, loss of control over intake, and the emergence of a negative emotional state during withdrawal (Koob and Volkow, 2010). Similar to stress, alcohol intake activates the hypothalamic-pituitary-adrenal (HPA) axis to release cortisol/corticosterone (CORT; cortisol in humans and corticosterone in rodents) from the adrenal gland (Ellis, 1966;

Received Jan. 5, 2012; revised April 10, 2012; accepted April 11, 2012.

Author contributions: L.F.V., E.P.Z., M.H., and G.F.K. designed research; L.F.V., E.B., J.E.S., K.K.M., T.W.W., M.L.L., V.R.-C., and P.P.S. performed research; L.F.V., E.B., C.R., V.R.-C., E.P.Z., P.P.S., M.H., and G.F.K. analyzed data; L.F.V., E.P.Z., M.H., and G.F.K. wrote the paper.

This is publication number 21284 from The Scripps Research Institute. This work was financially supported by National Institutes of Health Grant DA004043 from the National Institute on Drug Abuse, Grant GM085659 from National Institute of General Medicine, Grants AA006420, AA017371, AA012602, and AA013191 from the National Institute on Alcohol Abuse and Alcoholism (NIAAA), and the Pearson Center for Alcoholism and Addiction Research. Training was provided for authors J.E.S. and T.W.W. by NIAAA Grant T32AA007456, and for author M.L.L. by NIAAA Grant F32AA018914. We thank Michael Arends for proofreading the manuscript, Dr. Scott Edwards for critical comments regarding the manuscript, and Ashley Juniper, Daniel Ramirez, Zach Roy, Amand Srivastara, Imran Damany, Natalie Varnay, and Alexander Le for help with the behavioral experiments.

The authors declare no competing financial interests.

Correspondence should be addressed to Dr. Leandro F. Vendruscolo, Committee on the Neurobiology of Addictive Disorders, The Scripps Research Institute, 10550 North Torrey Pines Road, SP30 -2400, La Jolla, CA 92037. E-mail: leandro@scripps.edu.

DOI:10.1523/JNEUROSCI.0069-12.2012

Copyright $\odot 2012$ the authors $\quad 0270-6474 / 12 / 327563-09 \$ 15.00 / 0$
Lee and Rivier, 1997; Richardson et al., 2008). CORT contributes to the reinforcing effects of drugs (Fahlke et al., 1995, 1996; Goeders, 1997; Piazza and Le Moal, 1998; Mantsch et al., 1998; Uhart and Wand, 2009). Upon release, CORT binds to two types of brain receptors: mineralocorticoid receptors (MRs or type I, which have high affinity for CORT) and glucocorticoid receptors (GRs or type II, which have a lower affinity for CORT and are activated predominantly at high circulating CORT levels; McEwen et al., 1968, McEwen, 2007). A consequence of high CORT levels is the dysregulation of gene transcription, including corticotropin-releasing factor (CRF), a critical factor in alcohol dependence-related neuroadaptations (Heilig and Koob, 2007).

In alcohol dependence, the neuroendocrine stress system is dysregulated in both humans (Adinoff et al., 1990; 2003; Lovallo et al., 2000; Uhart and Wand, 2009; Sinha et al., 2011) and rodents (Rasmussen et al., 2000; Zorrilla et al., 2001; Richardson et al., 2008), but the consequences of this dysregulation for the escalation of alcohol intake, compulsive use, and relapse remain unclear. Following a prolonged history of alcohol dependence, negative reinforcement becomes a dominant motivational factor for continued alcohol use (i.e., alcohol is used to alleviate or prevent negative emotional symptoms, such as anxiety, dysphoria, and hypohedonia that emerge in the absence of the drug; Edwards and Koob, 2010). The transition from positive reinforcement in nondependent individuals to negative reinforce- 
ment in alcohol dependence may therefore be driven by dysregulated HPA axis function, yet the role of MRs and GRs in the transition to alcohol dependence remains to be investigated.

We tested the hypothesis that CORT receptors are involved in the escalated and compulsive alcohol intake that results when alcohol dependence is induced by alcohol vapor exposure. Compulsive drinking was assessed by a progressive-ratio (PR) test, in which the "price" (number of lever presses) required for the next reinforcement increases progressively (Hodos, 1961), and by a quinine adulteration test that measures persistent alcohol consumption despite the aversive bitter taste of quinine (added to the alcohol solution; Wolffgramm and Heyne, 1995). We found that acute alcohol withdrawal and protracted alcohol abstinence were associated with changes in glucocorticoid receptor (GR) expression levels in several stress/reward-related brain areas. Chronic GR antagonism prevented the development of escalated and compulsive alcohol drinking produced by alcohol vapor exposure during acute withdrawal and blocked the escalated and compulsive alcohol drinking during protracted abstinence in animals with a history of alcohol dependence. Altogether, the results suggest a critical role for GRs in the development and maintenance of escalated drinking in alcohol dependence.

\section{Materials and Methods}

Subjects. Adult male Wistar rats (Charles River), weighing 225-275 g at the beginning of the experiments, were housed in groups of 2-3 per cage in a temperature-controlled $\left(22^{\circ} \mathrm{C}\right)$ vivarium on a $12 \mathrm{~h} / 12 \mathrm{~h}$ light/dark cycle (lights on at 8:00 P.M.) with ad libitum access to food and water. All behavioral tests were conducted during the dark phase of the light/dark cycle. All procedures adhered to the National Institutes of Health Guide for the Care and Use of Laboratory Animals and were approved by the Institutional Animal Care and Use Committee of the Scripps Research Institute.

Operant self-administration. Self-administration sessions were conducted in standard operant conditioning chambers (Med Associates). The rats were first trained to self-administer alcohol using a modified (Walker and Koob, 2007) sucrose-fading procedure (Samson, 1986), in which $10 \%(\mathrm{w} / \mathrm{v})$ alcohol was added to a sweet solution and then sweeteners were gradually removed from the experimental solution. Upon completion of this procedure, the animals were allowed to selfadminister a $10 \%(\mathrm{w} / \mathrm{v})$ alcohol solution and water on a fixed-ratio 1 (FR1) schedule of reinforcement (i.e., each operant response was reinforced with $0.1 \mathrm{ml}$ of the solution). For the pharmacological tests with mifepristone, rats were trained to self-administer alcohol according to the following protocol that generated the same amount of baseline drinking but with less testing in preliminary studies. First, the rats were given free-choice access to alcohol $(10 \% \mathrm{w} / \mathrm{v})$ and water for $1 \mathrm{~d}$ in their home cages to habituate them to the taste of alcohol. Second, the rats were subjected to an overnight session in the operant chambers with access to one lever (right lever) that delivered water (FR1). Food was available ad libitum during this training. Third, after $1 \mathrm{~d}$ off, the rats were subjected to a $2 \mathrm{~h}$ session (FR1) for $1 \mathrm{~d}$ and a $1 \mathrm{~h}$ session (FR1) the next day, with one lever delivering alcohol (right lever). All of the subsequent sessions lasted $30 \mathrm{~min}$, and two levers were available (left lever: water; right lever: alcohol) until stable levels of intake were reached. Responding obtained with this procedure is equivalent to the sucrose-fading procedure (Samson, 1986; Walker and Koob, 2007). Upon completion of this procedure, the animals were allowed to self-administer a $10 \%(\mathrm{w} / \mathrm{v})$ alcohol solution and water on an FR1 schedule of reinforcement.

Alcohol vapor chambers. The rats were made dependent by chronic, intermittent exposure to alcohol vapors as previously described (O'Dell et al., 2004; Gilpin et al., 2008). They underwent cycles of $14 \mathrm{~h}$ on (blood alcohol levels during vapor exposure ranged between 150 and $250 \mathrm{mg} \%$ ) and $10 \mathrm{~h}$ off, during which behavioral testing for acute withdrawal occurred (i.e., 6-8 h after vapor was turned off when brain and blood alcohol levels are negligible; Gilpin et al., 2009). In this model, rats exhibit somatic withdrawal signs and negative emotional symptoms reflected by anxiety-like responses and elevated brain reward thresholds (Schulteis et al., 1995; Roberts et al., 2000; Valdez et al., 2002; Rimondini et al., 2003; O'Dell et al., 2004; Zhao et al., 2007; Sommer et al., 2008; Edwards et al., 2012). Nondependent rats were not exposed to alcohol vapor. For protracted abstinence, the animals were tested at the same time of the day as for acute withdrawal but 3-6 weeks after the vapor was turned off.

Operant self-administration during alcohol vapor exposure. Behavioral testing occurred 2-4 times per week. The rats were tested for alcohol (and water) self-administration on an FR1 schedule of reinforcement for 13 30-min sessions. Operant self-administration on an FR1 schedule requires minimal effort by the animal to obtain the reinforcer and herein was considered a measure of intake. For five sessions, the rats were tested on a PR schedule, under which the number of lever presses necessary to obtain the next reinforcer progressively increased according to the following progression: $1,1,2,2,3,3,4,4,5,5,7,7,9,9,11,11,13$, 13, etc. The PR session stopped after $90 \mathrm{~min}$ or when $15 \mathrm{~min}$ had elapsed without the rat obtaining a reinforcer. In this test, the workload ("price") for the next alcohol reinforcer increases progressively until the rat reaches a "breakpoint" (i.e., a measure of motivation/compulsivity) beyond which it no longer responds for alcohol.

The rats were then maintained on an FR1 schedule until stable levels of alcohol self-administration were reattained, and the alcohol solution was adulterated with increasing concentrations of quinine $(0.0005,0.001$, 0.0025 , and $0.005 \mathrm{~g} / \mathrm{L}$ ) presented between-sessions (one concentration per session). This test measures the persistence of animals to consume alcohol despite the aversive bitter taste of quinine that was added to the alcohol solution and was considered herein a measure of compulsive intake.

Finally, the rats were tested for saccharin $(0.004 \%$, w/v) selfadministration under an FR1 schedule for five 30 min sessions to determine whether the effects were specific for alcohol or generalized to other types of reward. A submaximal rewarding saccharin concentration was chosen based on previous studies (Vendruscolo et al., 2010) to prevent reaching a "ceiling effect" in any group and maintain similar response rates as alcohol.

Brain collection. Brains from dependent and nondependent rats were collected and snap-frozen with isopentane for measurements of GR and MR mRNA levels during acute alcohol withdrawal (24 h after the vapor was turned off). This time point was chosen because we were interested in more stable dysregulations of gene expression and to avoid any transient effects caused by earlier withdrawal. Importantly, the escalation of alcohol consumption has been demonstrated in rats at $2-8 \mathrm{~h}$ and $24 \mathrm{~h}$ of withdrawal and 2-7 weeks post-vapor (Valdez et al., 2002; Rimondini et al., 2003; O’Dell et al., 2004; Gilpin et al., 2008; Sommer et al., 2008). Brains from a separate cohort of animals were dissected 3 weeks after the vapor was turned off to investigate whether changes in GR mRNA levels could be detected during protracted alcohol abstinence. The brains were sliced on a cryostat, and bilateral punches $(300 \mu \mathrm{m}$ thickness, $2 \mathrm{~mm}$ diameter) were collected from the prefrontal cortex (PFC), nucleus accumbens (NAc), bed nucleus of the stria terminalis (BNST), amygdala, and hippocampus. Because we expected that smaller gene expression changes might occur during protracted alcohol abstinence, we dissected subregions of some of the regions used above for GR quantification from $300 \mu \mathrm{m}$ cryostat-cut slices (Cuello and Carson, 1983).

Quantitative nuclease protection array. The expression of GR and MR mRNA during acute alcohol withdrawal was assessed using quantitative nuclease protection assays (qNPA, High Throughput Genomics). This assay was used because it allowed us to quantitatively measure several stress/ reward-related genes at the same time. The primer sequences were the following: GR (position 85, GACTTTTATAAAAGCCTGAGGGGAGGAGCT ACA GTCAAGGTTTCTGCATC; position 318, GGGGCTGTATATGGGA GAGACAGAAACAAAAGTGATGGGGAATGACTTGG; position 1474, CCAGCATGCCGCTATCGGAAATGTCTTCAGGCTGGAATGAACCTT GAAGC; position 1734, CACACTCAACA TGTTAGGTGGGCGTCAAGT GATTGCAGCAGTGAAATGGC), MR (position 346, CCTCTCCATCCT CATTGCCGATCAGCCAGTATTGGACTTGCTGGTAGCGG; position 1764, GTCAAGCAAGCACTCATGTTCAGGCGCCTCTTTTAAAGGGA ACC CCACGG; position 3380, CTGGGAATGCCAAACCCCTTTACTTT CACAGAAAGTG ACGGGAGATACCG; position 4508, CCCGCGTGGGA 
AGTGTTCGTGAGACTCTAGTGCGCAGCTGTGGCTTCTGC). RNA was extracted and purified from brain tissue using the PureLinkTM RNA Mini Kit (Ambion) according to the manufacturer's instructions. Two hundred nanograms of total RNA were diluted in lysis buffer (up to $25 \mu \mathrm{l}$ ) that contained the cDNA riboprobes complementary to targeted RNA and added to a 96 -well plate. The plate was then heated at $95^{\circ} \mathrm{C}$ for $15 \mathrm{~min}$ (denaturation) and incubated for $16 \mathrm{~h}$ at $60^{\circ} \mathrm{C}$ for hybridization of the probes to RNA. The single-strand nucleic acids (unbound RNA and probes) were eliminated by S1-nuclease digestion for $90 \mathrm{~min}$ at $50^{\circ} \mathrm{C}$. The enzymatic reaction was terminated by adding $10 \mu \mathrm{l}$ of S1 stop solution, followed by incubation at $95^{\circ} \mathrm{C}$ for $15 \mathrm{~min}$ to inactivate the $\mathrm{S} 1$ nuclease and hydrolyze bound RNA. Ten microliters of neutralizing solution were added to hydrolyze the DNA:RNA heteroduplexes and degrade the RNA, leaving the sample with the selected probe only. The samples were then transferred for RNA quantification to a plate, in which 16 spots, each containing a linker to specifically capture each probe, had been printed into each well, and incubated at $50^{\circ} \mathrm{C}$ for $24 \mathrm{~h}$ to allow for probe hybridization to the plate. After several washes, $40 \mu \mathrm{l}$ of detection linker solution was added to each well, and the ArrayPlate was incubated at $60^{\circ} \mathrm{C}$ for $90 \mathrm{~min}$ to allow the detection linker to hybridize to the ArrayPlate. The plate was washed for another cycle and incubated at $37^{\circ} \mathrm{C}$ for 30 min after the addition of the detection enzyme (40 $\mu \mathrm{l})$. This step was followed by another wash and the addition of horseradish peroxidase chemiluminescent substrate. The chemiluminescent signal from each well of the ArrayPlate was quantified and reported by SuperCapella Imager.

Reverse transcription and quantitative PCR. Given that GR mRNA levels and not MR mRNA levels were differentially expressed in dependent and nondependent rats during acute alcohol withdrawal, we only measured GR mRNA levels during protracted alcohol abstinence. Total RNA was extracted using the PicoPure RNA Isolation kit (Applied Biosystems) and treated with DNase I (Qiagen). Concentrations were determined using the Quant-iT RiboGreen RNA Assay Kit (Invitrogen). cDNA was reverse-transcribed from total RNA using iScript cDNA (Bio-Rad) in the presence of Oligo (dT) and random primers according to the manufacturer's instructions. Gene expression levels were determined by quantitative PCR (qPCR) using a SYBR Green-based detection system (iQ SYBR Green Supermix, Bio-Rad Laboratories). Reactions were performed on laser-equipped thermal cyclers to detect changes in fluorescence in real time, and cDNA concentrations of GR were calculated according to the relative quantification (ddCt) method, corrected for differences in PCR efficiency, and normalized to glyceraldehyde-3-phosphate dehydrogenase (Gapdh), cyclophilin A (Ppia), or $\beta$-actin $(A c t b)$. The following primers were used: GR primer pair 1 (forward, $5^{\prime}$ TACAAAGATTGCAGGTATCCTATGA 3'; reverse, $5^{\prime}$ ACTCTT GGCTCTTCAGACCTTC $3^{\prime}$ ), primer pair 2 (forward, 5' GCACCAGCTA TCAGAAGACC 3'; reverse, 5' GCTCTACACCAGTTAGGACG 3'), Ppia (forward, 5' TATCTGCACTGCCAAGACTGAGTG 3'; reverse, 5' CTTCTT GCTGGTCTTGCCATTCC 3'), Actb (forward, 5' AGATTACTGCCCT GGCTCCT 3'; reverse, 5' CAGTGAGGCCAGGATAGAGC 3').

Mifepristone (RU38486) treatment. To investigate the functional role of GRs in the escalation of alcohol self-administration during alcohol vapor exposure, the rats were trained to self-administer alcohol as described above and subcutaneously implanted with mifepristone pellets (a GR/progesterone receptor antagonist; $150 \mathrm{mg}$; Innovative Research of America) or placebo pellets for chronic release $(21 \mathrm{~d})$. The mifepristone dose was chosen based on previous studies (Schneider et al., 2003; Nephew et al., 2008) and adjusted for bodyweight. Twenty-four hours later, alcohol vapor exposure and behavioral testing began. To investigate the functional role of GRs in escalated alcohol self-administration during protracted alcohol abstinence, additional groups of dependent and nondependent rats were subcutaneously implanted with mifepristone or placebo pellets 1 week after the vapor was turned off. Behavioral testing began 1 week after pellet implantation. Three animals from this experiment were excluded: one mifepristone-treated dependent rat and one placebo-treated nondependent rat that showed rejection of the pellet and one placebo-treated dependent rat outlier.

Statistical analysis. The data are expressed as mean and SEM. The data were analyzed using ANOVA with or without repeated measures, with session as the within-subjects factor and group (dependent vs nondependent) and treatment (mifepristone vs placebo pellet) as between-subjects factors. When appropriate, post hoc comparisons were performed using
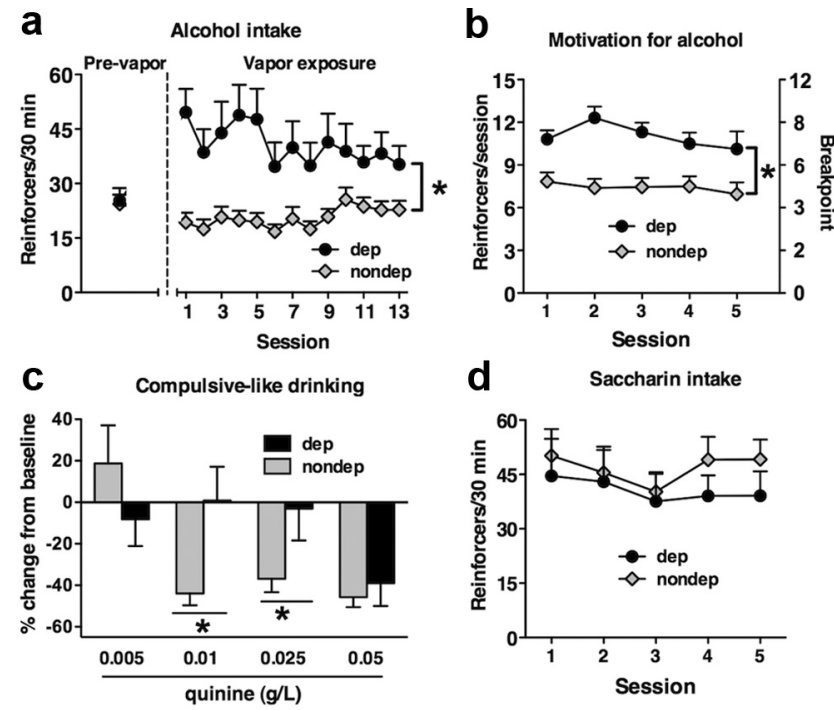

Figure 1. Specific increase in alcohol intake and compulsive drinking in alcohol vaporexposed rats during acute alcohol withdrawal. $\boldsymbol{a}$, Number of lever presses for alcohol before (pre-vapor) and during alcohol vapor exposure on a fixed-ratio 1 (FR1) schedule of reinforcement (i.e., every active lever press was reinforced with $0.1 \mathrm{ml}$ of $10 \%$ alcohol, w/v). $\boldsymbol{b}$, Number of alcohol reinforcers earned and last ratio achieved in a progressive-ratio test. c, Compulsivelike drinking (i.e., persistent alcohol drinking despite the aversive bitter taste of quinine added the alcohol solution). The data represent the percentage change from baseline (i.e., lever presses for alcohol alone before adulteration with quinine). $\boldsymbol{d}$, Number of lever presses for a saccharin $(0.004 \%, w / v)$ solution (FR1). The data represent mean and SE. ${ }^{*} p<0.05$, significant difference between dependent and nondependent. $n=16-18$ per group.

Fisher's Least Significant Difference (LSD) test. Glucocorticoid receptor and MR mRNA levels were compared using Student's $t$ test. The accepted level of significance for all tests was $p \leq 0.05$.

\section{Results}

\section{Alcohol vapor-exposed animals show increased alcohol} intake, motivation for alcohol, and compulsive drinking

Pre-vapor operant responding for alcohol is shown in Figure 1a. Subsequent testing began after 1 month of intermittent alcohol vapor exposure ("dependence induction" for the dependent group) and was performed during acute withdrawal (6-8 h after the vapor was turned off). Vapor-exposed dependent rats showed increased lever press responding for alcohol (group effect: $F_{(1,32)}=7.6, p<0.01$ ) compared with nondependent rats (Fig. 1a). Similar results were obtained for alcohol intake (in $\mathrm{g} / \mathrm{kg}$ ). With the exception of session 10 , dependent rats $(0.68 \pm 0.08 \mathrm{~g} / \mathrm{kg} / 30 \mathrm{~min})$ showed higher intake than nondependent rats $(0.3 \pm 0.02 \mathrm{~g} / \mathrm{kg} / 30 \mathrm{~min})$ in all self-administration sessions (group $\times$ session interaction: $F_{(12,384)}=2.8, p<0.001 ; \mathrm{LSD}$ post hoc test: $p<0.05)$. Dependent rats also showed increased responding compared with nondependent rats (group effect: $\left.F_{(1,32)}=17.1, p<0.0005\right)$ in the PR test (Fig. $1 b$ ).

For the quinine-adulteration test (Fig. 1c), the analyses revealed a significant group $\times$ concentration interaction $\left(F_{(3,96)}=\right.$ 5.6, $p<0.001)$ for the percentage change from baseline consumption. Dependent rats showed more resistance to the aversive taste of quinine, with significantly greater intake by dependent rats than nondependent rats of solutions adulterated with 0.01 $\mathrm{g} / \mathrm{L}$ quinine $(p<0.01)$ and $0.025 \mathrm{~g} / \mathrm{L}$ quinine $(p<0.05)$. Because quinine may be appetitive at low concentrations (Da Silva et al., 2005), and a trend toward increased drinking was observed in nondependent rats at the lowest quinine concentration, we additionally performed a statistical analysis that excluded the lowest 
quinine concentration. The results again revealed a significant group $\times$ concentration interaction $\left(F_{(2,64)}=5.0, p<0.01\right)$, thus confirming the robustness of the observed effect.

To control for taste differences between groups, another set of rats was given quinine solution $(0.025 \mathrm{~g} / \mathrm{L})$ without alcohol. A $36.6 \%$ versus $32.9 \%$ reduction in responding was observed in vapor-exposed versus control rats $(p=0.8)$, respectively, compared with baseline responding for alcohol. When $10 \%$ alcohol was added to the quinine solution, vapor-exposed animals increased their intake to the previous levels of alcohol self-administration, whereas control animals showed even lower levels of alcohol/quinine intake (group effect: $p<$ 0.01). Thus, dependent and nondependent rats were equally sensitive to the aversive bitter taste of quinine, but only dependent rats were "motivated" to overcome the aversiveness of quinine to obtain alcohol.

The increased alcohol intake, motivation for alcohol consumption, and compulsive drinking exhibited by dependent rats was specific for alcohol because dependent and nondependent rats did not differ in the selfadministration of saccharin-sweetened water (Fig. 1d).

\section{Alcohol withdrawal is associated with GR mRNA expression changes in stress/ reward-related brain regions}

We next determined GR and MR mRNA expression levels in several stress/rewardrelated brain regions. Acute alcohol withdrawal (24 h after the alcohol vapor was turned off) was associated with GR mRNA downregulation in the PFC $\left(t_{20}=2.1, p<0.05\right.$; Fig. $2 a), \operatorname{NAc}\left(t_{18}=2.7, p<0.05\right.$; Fig. $\left.2 b\right)$, and BNST $\left(t_{24}=2.7, p<0.05\right.$; Fig. $2 c$ ) but not amygdala (Fig. $2 d$ ) or hippocampus (Fig. $2 e$ ). No group differences were found for MR mRNA levels in any brain areas.

For protracted alcohol abstinence ( 3 weeks after the alcohol vapor was turned off), we found that GR mRNA was significantly higher in dependent rats than in nondependent rats in the NAc core $\left(t_{12}=3.6, p<0.005\right)$, ventral BNST $\left(t_{10}=2.6, p<0.05\right)$, and central nucleus of the amygdala $\left(\mathrm{CeA} ; t_{11}=4.4, p<0.005\right)$ but not PFC, NAc shell, dorsolateral BNST, or basolateral nucleus of the amygdala (BLA; Fig. 3).

\section{Antagonism of GRs prevents the development of alcohol dependence and blocks escalated alcohol intake during protracted abstinence}

Before vapor exposure, the groups selected as dependent and nondependent did not differ significantly with regard to baseline alcohol intake. Chronic mifepristone treatment selectively blocked the escalation of alcohol intake in vapor-exposed rats (group $\times$ treatment $\times$ day interaction: $\left.F_{(4,136)}=2.8, p<0.05\right)$. Placebo-treated, vapor-exposed rats exhibited a significant increase in lever pressing for alcohol compared with placebotreated nondependent rats on day $10(p<0.05)$, day $13(p<$ $0.005)$, and day $17(p<0.05)$ of vapor exposure and compared b

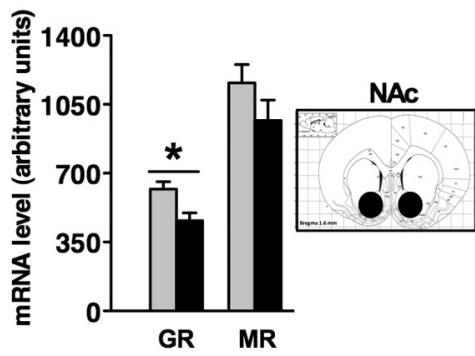

d

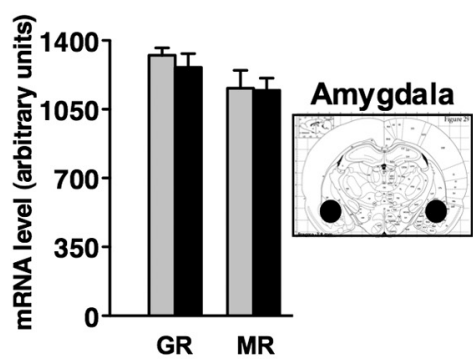

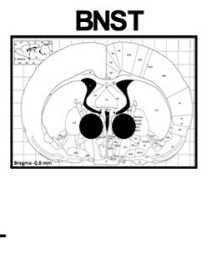

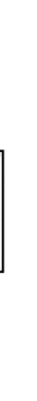
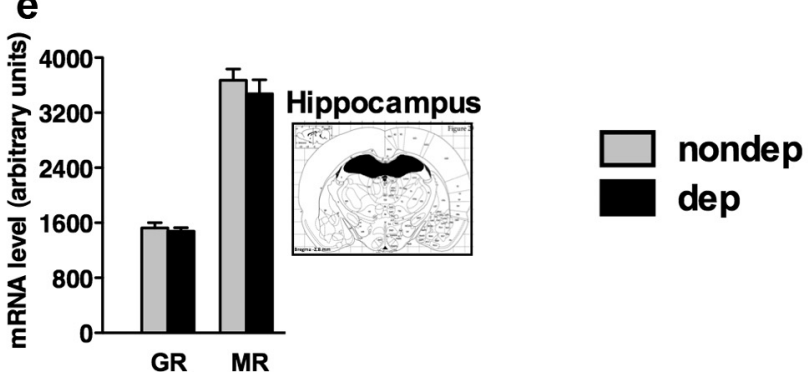

Figure 3. Protracted alcohol abstinence (3 weeks after the vapor was turned off) was accompanied by GR upregulation in stress/reward-related brain areas. Dependent rats showed higher GR mRNA levels in the NAc shell, ventral BNST, and central nucleus of the amygdala (CeA) but not PFC, NAc core, dorsolateral (DL) BNST, or basolateral amygdala (BLA) compared with nondependent rats. The data represent mean and SE. ${ }^{*} p<0.05$, significant difference between dependent and nondependent. $n=5-8$ per group.

with mifepristone-treated vapor-exposed rats on day $6(p<$ $0.05)$, day $10(p<0.005)$, day $13(p<0.001)$, and day $17(p<$ $0.001)$ of vapor exposure. Mifepristone-treated, vapor-exposed rats did not differ from nondependent rats, and mifepristone did not affect lever pressing for alcohol in nondependent rats (Fig. $4 b)$. Although a marginal, nonsignificant effect was detected for alcohol intake (in $\mathrm{g} / \mathrm{kg}$; treatment $\times$ group $\times$ session interaction: $\left.F_{(4,136)}=2.2, p=0.07\right)$, additional analyses performed separately by group revealed an overall treatment effect in dependent rats only $\left(F_{(5,85)}=3.3, p<0.01\right)$, with mifepristone-treated dependent rats displaying lower alcohol intake compared with placebo- 
a

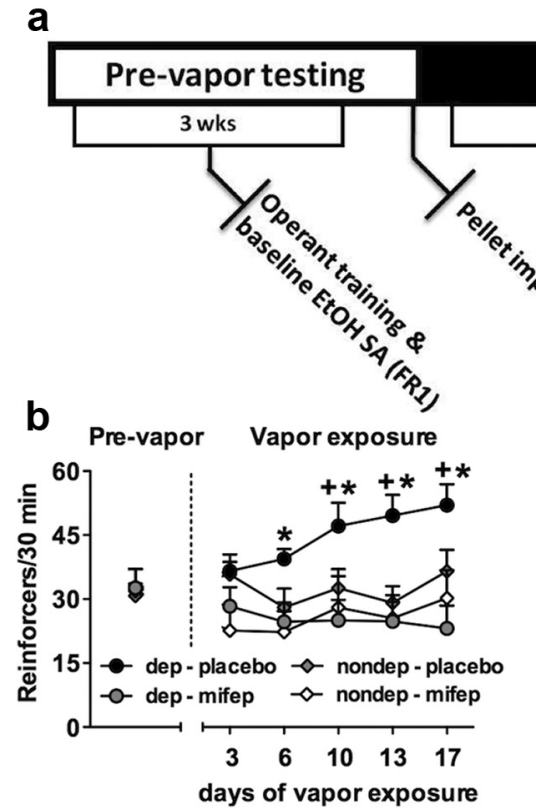

Figure 4. Chronic GR blockade by mifepristone prevented the escalation of alcohol intake and motivation for alcohol in vaporexposed animals during acute alcohol withdrawal. $\boldsymbol{a}$, Timeline of the experiment. Dependent and nondependent rats were implanted with pellets for the chronic release of the GR antagonist mifepristone (150 $\mathrm{mg}$ for $21 \mathrm{~d}$ ) or placebo before exposure to alcohol vapor. Mifepristone-treated vapor-exposed rats did not exhibit an escalation of alcohol intake $(\boldsymbol{b})$ or increased PR responding (c) compared with placebo-treated vapor-exposed rats. Mifepristone did not influence alcohol intake in nondependent rats. The data represent mean and SE. ${ }^{*} p<0.05$, significant difference from mifepristone-treated vapor exposed rats; ${ }^{+} p<0.05$, significant difference from placebo-treated nondependent rats. $n=9-10$ per group.

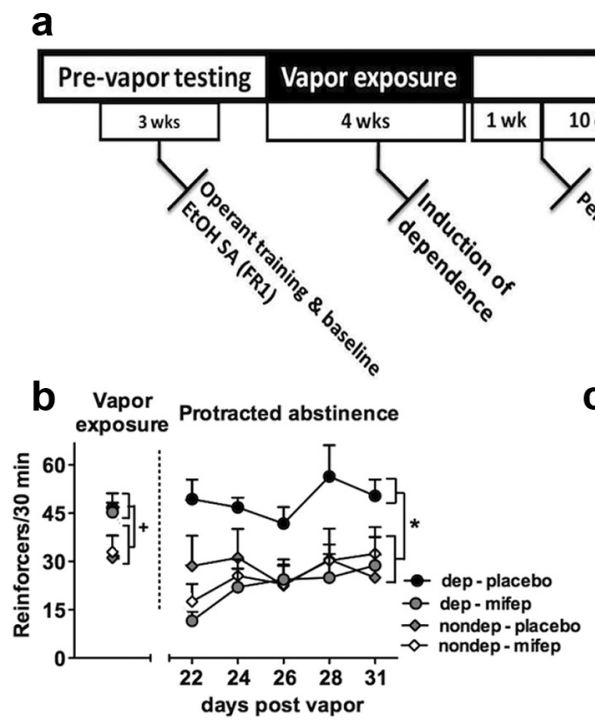

\section{C}

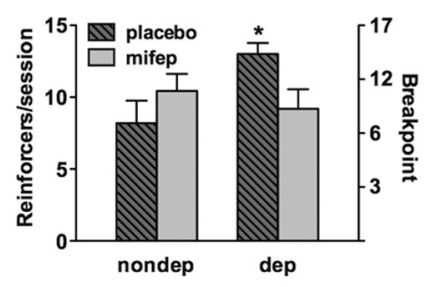

Figure 5. Chronic GR blockade by mifepristone decreased escalated alcohol intake in vapor-exposed animals during protracted alcohol abstinence. $\boldsymbol{a}$, Timeline of the experiment. The rats were made dependent on alcohol by exposure to chronic, intermittent vapor exposure and then removed from the vapor chambers. One week later, dependent and nondependent rats were implanted with pellets for the chronic release of the GR antagonist mifepristone ( $150 \mathrm{mg}$ for $21 \mathrm{~d}$ ) or placebo. Behavioral testing began 1 week after pellet implantation (3 weeks of protracted withdrawal). Mifepristone-treated vapor-exposed rats did not exhibit escalated alcohol intake $(\boldsymbol{b})$ or increased PR responding (c) compared with placebo-treated vapor-exposed rats. Mifepristone did not influence alcohol intake in nondependent rats. The data represent mean and SE. ${ }^{*} p<0.05$, significant different from mifepristonetreated dependent rats and placebo-treated nondependent rats. $n=5-7$ per group.

treated dependent rats. The average intake was $0.51 \pm 0.12$ versus $0.98 \pm 0.08 \mathrm{~g} / \mathrm{kg} / 30 \mathrm{~min}$ for mifepristone- and placebo-treated dependent rats, respectively.

For the PR test, a group $\times$ treatment interaction $\left(F_{(1,34)}=6.1\right.$, $p<0.05)$ was detected. Mifepristone blocked the increased re- sponding for alcohol produced by alcohol vapor exposure $(p<0.005)$, without altering responding to obtain alcohol in nondependent controls (Fig. 4c).

The results for the effect of mifepristone on alcohol self-administration during protracted alcohol abstinence are shown in Figure 5. During alcohol vapor exposure, dependent rats displayed higher alcohol self-administration compared with nondependent rats $\left(F_{(1,18)}=\right.$ 6.6, $p<0.05)$. Placebo-treated rats with a history of alcohol dependence displayed escalated alcohol intake during protracted abstinence, and mifepristone treatment blocked this effect. The treatment did not affect alcohol intake in nondependent rats. Indeed, placebo-treated dependent rats displayed higher alcohol intake compared with all of the other groups (group vs treatment interaction: $F_{(1,18)}=5.1, p<0.05$; LSD post hoc test: $p<0.05)$.

For the PR test, a group $\times$ treatment interaction $\left(F_{(1,18)}=5.7, p<0.05\right)$ was detected. Placebo-treated dependent rats displayed increased responding for alcohol compared with mifepristone-treated dependent rats and placebo-treated nondependent rats ( $p<0.05$; Fig. $5 c)$.

\section{Discussion}

We report here that rats made dependent on alcohol by chronic, intermittent alcohol vapor exposure displayed a specific increase in alcohol intake and compulsive alcohol drinking compared with nondependent rats, traits that are thought to be hallmarks of alcohol dependence. Compared with nondependent rats, dependent rats showed GR mRNA downregulation in several stress/reward-related brain areas during acute withdrawal and GR upregulation during protracted alcohol abstinence. A functional role for GRs in alcohol dependence was demonstrated by showing that chronic GR blockade during the course of alcohol vapor exposure prevented the escalation of alcohol intake and blocked the increase in PR responding. Chronic GR antagonism also blocked escalated and compulsive alcohol drinking during protracted abstinence in rats with a history of alcohol dependence. These results suggest a critical role for GR in the development and maintenance of alcohol dependence.

\section{Alcohol withdrawal-associated GR mRNA changes in stress/reward-related brain regions \\ Glucocorticoid receptor mRNA was} downregulated in the PFC, NAc, and BNST during acute alcohol withdrawal, an effect also observed with chronic stress (de Kloet et al., 2005; Noguchi et al., 2010). The lack of effect in the amygdala and hippocampus does not exclude the possibility of 


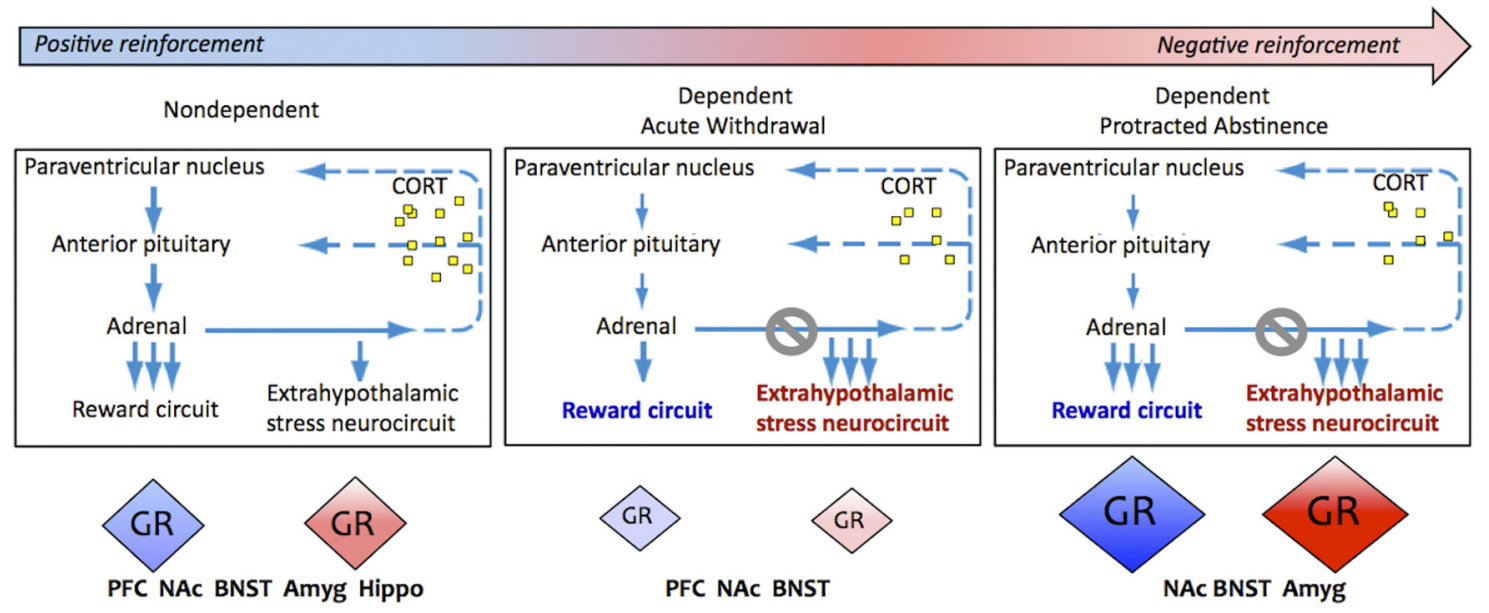

Figure 6. Hypothalamic-pituitary-adrenal axis, extrahypothalamic GR levels, and brain stress/reward function hypothesized to be recruited at different stages of the addiction cycle as addiction moves from positive reinforcement to negative reinforcement. Left, In nondependent subjects, alcohol activates the HPA axis to release CORT from the adrenal gland (Ellis, 1966; Lee and Rivier, 1997; Richardson et al., 2008), and CORT facilitates the reinforcing effects of alcohol (Fahlke et al., 1995, 1996) via positive reinforcement. High CORT levels decrease HPA axis activity via negative feedback. Middle, Repeated cycles of alcohol intoxication/withdrawal induce overactivation of the HPA axis that disrupt HPA axis function (i.e., blunted activity; Richardson et al., 2008) and downregulate GRs levels in stress/reward-related brain regions. These long-lasting changes can "sensitize" extrahypothalamic stress systems (e.g., (RF) involved in the behavioral response to stressors and further drive escalated and compulsive drug intake (Makino et al., 2002) via negative reinforcement (Edwards and Koob, 2010). Right, During protracted abstinence, GR levels are upregulated in stress/reward-related brain regions, suggesting receptor adaptation when alcohol vapor exposure ceases. Although peak HPA axis activation is blunted during protracted abstinence in alcohol dependence (Adinoff et al., 1990; Zorrilla et al., 2001), alcohol dependence-mediated dysregulation of GRs remains. A sensitized GR system would be expected to increase the "gain" for a neuronal response to CORT, sustaining the escalation of alcohol intake even in the absence of peak levels of released CORT. There are several steps between GR binding, mRNA expression, and function. Pinpointing the single molecular mechanism that underlies escalated alcohol intake is difficult at this time. The bidirectional regulation of GRs at different withdrawal time-points suggests that GR expression is dynamically regulated in the alcohol-dependent and postdependent brain and mediates escalated alcohol drinking. Amyg, amygdala; Hippo, hippocampus.

alterations in GR levels in these brain regions because the measurements included the whole structures rather than discrete subregions. In contrast, GR levels were increased in the NAc core, $\mathrm{CeA}$, and ventral BNST during protracted alcohol abstinence, suggesting receptor adaptation when alcohol exposure ceased. This bidirectional regulation of GRs at different withdrawal timepoints suggests that GR expression is dynamically regulated in the alcohol-dependent and postdependent brain. Evidence shows that both increased GR activity and decreased GR activity are associated with increased anxiety (Wei et al., 2004; Ridder et al., 2005; Jakovcevski et al., 2008), but escalated alcohol intake during protracted abstinence may also involve GR and reward system sensitization (Piazza and Le Moal, 1998). Thus, opposite changes in GR levels during acute alcohol withdrawal and protracted abstinence may play a role in sensitivity to stress/reward and escalated alcohol intake during these distinct phases of alcohol dependence (Valdez et al., 2002; Rimondini et al., 2003; O'Dell et al., 2004; Zhao et al., 2007; Gilpin et al., 2008; Sommer et al., 2008). Corticosterone levels have been shown to be blunted during acute withdrawal and protracted abstinence in our models of alcohol dependence (Zorrilla et al., 2001; Richardson et al., 2008), but directly relating CORT levels to GR changes during these phases would be an important future study. Altogether, the present findings suggest the occurrence of allostatic-like changes in the extrahypothalamic GR system in alcohol dependence.

Chronic GR antagonism blocks the escalation of alcohol intake during acute alcohol withdrawal and reverses escalated alcohol intake during protracted alcohol abstinence

Chronic mifepristone treatment during alcohol vapor exposure completely prevented the escalation of alcohol intake in vaporexposed rats, indicating that functional GRs are required for the escalation of alcohol intake that is associated with the development of alcohol dependence. Mifepristone did not affect alcohol consumption in nondependent rats, as previously reported
(Fahlke et al., 1995; 1996; O'Callaghan et al., 2005; Yang et al., 2008; Lowery et al., 2010). This result suggests that mifepristone prevents neuroplasticity involved in escalated alcohol drinking but does not influence the positive reinforcing properties of alcohol that are hypothesized to maintain moderate alcohol drinking in nondependent rats. Mifepristone also blocked the increased responding on a PR schedule produced by dependence, suggesting a decrease in the development of compulsive drinking. Progressive-ratio responding can be linked to the construct of compulsivity, in which responding to alcohol is persistent in the face of adverse consequences (i.e., increased cost for each subsequent reward) (Koob, 2012). Mifepristone has also been shown to attenuate behavioral sensitization produced by repeated stress or alcohol injections (Roberts et al., 1995), decrease alcohol drinking in limited-access conditions (Koenig and Olive, 2004), and reduce the stress-induced reinstatement of alcohol seeking (Simms et al., 2012) in nondependent rats, suggesting that mifepristone can affect alcohol-related behaviors under stressful conditions. Additionally, placebo-treated rats with a history of alcohol dependence drank more alcohol during protracted abstinence compared with nondependent rats, as previously reported (Valdez et al., 2002; Rimondini et al., 2003; Gilpin et al., 2008; Sommer et al., 2008). In the present study, chronic mifepristone treatment also decreased alcohol intake during protracted abstinence in rats with a history of alcohol dependence to the levels of nondependent rats. This effect was selective for alcohol dependence (i.e., nondependent alcohol drinking was unaffected by mifepristone). Possibly, low doses of mifepristone block the reinforcing effects of alcohol only when the GR and reward systems are hypersensitive, similar to protracted abstinence. These results support a functional role for GRs in escalated drinking during protracted abstinence.

Although peak HPA axis activation is blunted during protracted abstinence in alcohol dependence (Adinoff et al., 1990; Zorrilla et al., 2001), alcohol dependence-mediated GR dysregu- 
lation remains. A sensitized GR system would be expected to increase the "gain" for a neuronal response to CORT, sustaining the escalation of alcohol intake even in the absence of peak levels of released CORT. This sensitization of GR function may occur at several levels. One possibility is that there is a greater overall number of cytosolic GRs that facilitate ligand-dependent receptor dimerization and translocation to the nucleus. Alternatively, there may be altered phosphorylation of the GR (e.g., Sr203, Ser211, Ser226), thus modulating its transcriptional activity and altering its protein stability and subcellular location (Wallace and Cidlowski, 2001; Itoh et al., 2002; Miller et al., 2005; Chen et al., 2008; Avenant et al., 2010). Another possibility is that GR dissociation with cytosolic binding partners (HSPs, FKBPs) and nuclear translocation may be sensitized following repeated activation of these pathways. Additionally, the nuclear interactions between GR and other cofactors or transcriptional regulators (e.g., AP-1, NFKB) may remain altered during protracted abstinence and sensitize the GR system indirectly. Ligandindependent GR function (Eickelberg et al., 1999; Verhoog et al., 2011; Galliher-Beckley et al., 2011; Ritter et al., 2012) may also be sensitized. Each of these molecular processes may work to sensitize the GR system and maintain escalation despite blunted CORT release. However, given the several steps between GR binding, mRNA expression, and function (Reichardt and Schütz, 1998; Davies et al., 2008), pinpointing a single molecular mechanism that underlies escalated alcohol intake is difficult. Understanding this process will clearly require further research.

Mifepristone also inhibits the progesterone receptor (Peeters et al., 2004), which may participate in alcohol-related behaviors (Janis et al., 1998). However, the effects of mifepristone during alcohol withdrawal appear to be GR-specific (Jacquot et al., 2008). Moreover, one question is whether mifepristone can penetrate the blood-brain barrier. Mifepristone has somewhat limited blood-brain barrier permeability, but the concentrations attained in the brain are still significant (almost one-third those of serum; Heikinheimo and Kekkonen, 1993). Accordingly, systemic mifepristone administration produces behavioral changes (Roberts et al., 1995; Koenig and Olive, 2004; Simms et al., 2012), inhibits neurogenesis (Oomen et al., 2007), and, most directly, competes with dexamethasone for binding sites in the rat CNS (Allen et al., 1988). Our results likewise suggest that mifepristone reaches central GRs at concentrations sufficient for receptor occupancy.

Furthermore, CORT is released in hourly pulses with a higher amplitude of release during the active period (i.e., the dark in rodents), thus reflecting the circadian pattern of release (Young et al., 2004). Because of the relatively low affinity of CORT for GRs, these receptors are only activated during stressful conditions or during the circadian peaks of CORT release. Thus, chronic blockade of GRs by mifepristone in hypothalamic and extrahypothalamic structures may prevent GR activation and function during the high levels of CORT observed during alcohol intoxication/withdrawal, but chronic blockade may also influence the circadian rhythm of CORT release, which also can affect stress-related behaviors (Young et al., 2004; Sarabdjitsingh et al., 2010).

\section{Conclusions}

Previous work has shown that CORT facilitates the reinforcing effects of alcohol in nondependent subjects (Fahlke et al., 1995, 1996). However, high circulating levels of CORT during alcohol intoxication/withdrawal can feed back to shut off the HPA axis, "sensitize" extrahypothalamic stress systems (e.g., CRF) involved in the behavioral response to stressors, and further drive escalated and compulsive drug intake (Makino et al., 2002). Therefore, although activation of the HPA axis may contribute to the reinforcing effects of alcohol during initial drug use, its excessive activation may lead to the sensitization of brain stress systems that mediate negative reinforcement in the transition to alcohol dependence. The present study suggests that changes in GR function in extrahypothalamic stress systems, possibly via a hypothesized initial excessive activation of GRs, are involved in alcohol dependence (Fig. 6). Normalizing GR signaling early in the transition to dependence may block the sensitization of the brain stress systems, and the normalization of GR function after alcohol detoxification may reset the reward system to result in a shift from negative reinforcement to "normal" positive reinforcement or block the well documented sensitization of reward associated with protracted abstinence. Importantly, altered GR levels have been found in the superior frontal cortex of alcoholics (Liu et al., 2007; Ponomarev et al., 2012), and mifepristone has some promise for treating dysregulated mood (Flores et al., 2006; Nihalani and Schwartz, 2007; Blasey et al., 2009). The present results indicate that the GR system may be an attractive potential pharmacological target for the treatment of alcohol dependence.

\section{References}

Adinoff B, Martin PR, Bone GH, Eckardt MJ, Roehrich L, George DT, Moss HB, Eskay R, Linnoila M, Gold PW (1990) Hypothalamic-pituitaryadrenal axis functioning and cerebrospinal fluid corticotropin releasing hormone and corticotropin levels in alcoholics after recent and long-term abstinence. Arch Gen Psychiatry 47:325-330.

Adinoff B, Ruether K, Krebaum S, Iranmanesh A, Williams MJ (2003) Increased salivary cortisol concentrations during chronic alcohol intoxication in a naturalistic clinical sample of men. Alcohol Clin Exp Res 27:1420-1427.

Allen BD, Sutanto W, Jones MT (1988) A correlative study of RU38486 biopotency and competition with $[3 \mathrm{H}]$ dexamethasone for receptors in the rat central nervous system. J Steroid Biochem 30:411-415.

Avenant C, Kotitschke A, Hapgood JP (2010) Glucocorticoid receptor phosphorylation modulates transcription efficacy through GRIP-1 recruitment. Biochemistry 49:972-985.

Blasey CM, Debattista C, Roe R, Block T, Belanoff JK (2009) A multisite trial of mifepristone for the treatment of psychotic depression: a site-bytreatment interaction. Contemp Clin Trials 30:284-288.

Chen W, Dang T, Blind RD, Wang Z, Cavasotto CN, Hittelman AB, Rogatsky I, Logan SK, Garabedian MJ (2008) Glucocorticoid receptor phosphorylation differentially affects target gene expression. Mol Endocrinol 22:1754-1766.

Cuello A, Carson S (1983) Microdissection of fresh rat brain tissue slices. In: Brain microdissection techniques (Cuello A, ed), pp 37-126. New York: John Wiley.

Da Silva GE, Vendruscolo LF, Takahashi RN (2005) Effects of ethanol on locomotor and anxiety-like behaviors and the acquisition of ethanol intake in Lewis and spontaneously hypertensive rats. Life Sciences 77:693-706.

Davies L, Karthikeyan N, Lynch JT, Sial EA, Gkourtsa A, Demonacos C, Krstic-Demonacos M (2008) Cross talk of signaling pathways in the regulation of the glucocorticoid receptor function. Mol Endocrinol 22:1331-1344.

de Kloet ER, Joëls M, Holsboer F (2005) Stress and the brain: from adaptation to disease. Nat Rev Neurosci 6:463-475.

Edwards S, Koob GF (2010) Neurobiology of dysregulated motivational systems in drug addiction. Future Neurol 5:393-401.

Edwards S, Vendruscolo LF, Schlosburg JE, Misra KK, Wee S, Park PE, Schulteis G, Koob GF (2012) Development of mechanical hypersensitivity in rats during heroin and ethanol dependence: Alleviation by CRF(1) receptor antagonism. Neuropharmacology 62:1142-1151.

Eickelberg O, Roth M, Lörx R, Bruce V, Rüdiger J, Johnson M, Block LH (1999) Ligand-independent activation of the glucocorticoid receptor by beta2-adrenergic receptor agonists in primary human lung fibroblasts and vascular smooth muscle cells. J Biol Chem 274:1005-1010. 
Ellis FW (1966) Effect of ethanol on plasma corticosterone levels. J Pharmacol Exp Ther 153:121-127.

Fahlke C, Hård E, Eriksson CJ, Engel JA, Hansen S (1995) Consequence of long-term exposure to corticosterone or dexamethasone on ethanol consumption in the adrenalectomized rat, and the effect of type I and type II corticosteroid receptor antagonists. Psychopharmacology (Berl) 117: $216-224$.

Fahlke C, Hård E, Hansen S (1996) Facilitation of ethanol consumption by intracerebroventricular infusions of corticosterone. Psychopharmacology (Berl) 127:133-139.

Flores BH, Kenna H, Keller J, Solvason HB, Schatzberg AF (2006) Clinical and biological effects of mifepristone treatment for psychotic depression. Neuropsychopharmacology 31:628-636.

Galliher-Beckley AJ, Williams JG, Cidlowski JA (2011) Ligand-independent phosphorylation of the glucocorticoid receptor integrates cellular stress pathways with nuclear receptor signaling. Mol Cell Biol 31:4663-4675.

Gilpin NW, Richardson HN, Lumeng L, Koob GF (2008) Dependenceinduced alcohol drinking by alcohol-preferring $(\mathrm{P})$ rats and outbred Wistar rats. Alcohol Clin Exp Res 32:1688-1696.

Gilpin NW, Smith AD, Cole M, Weiss F, Koob GF, Richardson HN (2009) Operant behavior and alcohol levels in blood and brain of alcoholdependent rats. Alcohol Clin Exp Res 33:2113-2123.

Goeders NE (1997) A neuroendocrine role in cocaine reinforcement. Psychoneuroendocrinology 22:237-259.

Heikinheimo O, Kekkonen R (1993) Dose-response relationships of RU 486. Ann Med 25:71-76.

Heilig M, Koob GF (2007) A key role for corticotropin-releasing factor in alcohol dependence. Trends Neurosci 30:399-406.

Hodos W (1961) Progressive ratio as a measure of reward strength. Science 134:943-944.

Itoh M, Adachi M, Yasui H, Takekawa M, Tanaka H, Imai K (2002) Nuclear export of glucocorticoid receptor is enhanced by c-Jun N-terminal kinase-mediated phosphorylation. Mol Endocrinol 16:2382-2392.

Jacquot C, Croft AP, Prendergast MA, Mulholland P, Shaw SG, Little HJ (2008) Effects of the glucocorticoid antagonist, mifepristone, on the consequences of withdrawal from long term alcohol consumption. Alcohol Clin Exp Res 32:2107-2116.

Jakovcevski M, Schachner M, Morellini F (2008) Individual variability in the stress response of $\mathrm{C} 57 \mathrm{BL} / 6 \mathrm{~J}$ male mice correlates with trait anxiety. Genes Brain Behav 7:235-243.

Janis GC, Devaud LL, Mitsuyama H, Morrow AL (1998) Effects of chronic ethanol consumption and withdrawal on the neuroactive steroid 3alphahydroxy-5alpha-pregnan-20-one in male and female rats. Alcohol Clin Exp Res 22:2055-2061.

Koenig HN, Olive MF (2004) The glucocorticoid receptor antagonist mifepristone reduces ethanol intake in rats under limited access conditions. Psychoneuroendocrinology 29:999-1003.

Koob GF (2012) Theoretical frameworks and mechanistic aspects of alcohol addiction: alcohol addiction as a reward deficit disorder. In: Behavioral neurobiology of alcohol addiction (series title: Current topics in behavioral neuroscience, Vol 13) (Spanagel R, Sommer WH eds). New York: Springer.

Koob GF, Volkow ND (2010) Neurocircuitry of addiction. Neuropsychopharmacology 35:217-238.

Lee S, Rivier C (1997) An initial, three-day-long treatment with alcohol induces a long-lasting phenomenon of selective tolerance in the activity of the rat hypothalamic-pituitary-adrenal axis. J Neurosci 17:8856-8866.

Liu J, Lewohl JM, Harris RA, Dodd PR, Mayfield RD (2007) Altered gene expression profiles in the frontal cortex of cirrhotic alcoholics. Alcohol Clin Exp Res 31:1460-1466.

Lovallo WR, Dickensheets SL, Myers DA, Thomas TL, Nixon SJ (2000) Blunted stress cortisol response in abstinent alcoholic and polysubstanceabusing men. Alcohol Clin Exp Res 24:651-658.

Lowery EG, Spanos M, Navarro M, Lyons AM, Hodge CW, Thiele TE (2010) CRF-1 antagonist and CRF-2 agonist decrease binge-like ethanol drinking in C57BL/6J mice independent of the HPA axis. Neuropsychopharmacology 35:1241-1252.

Makino S, Hashimoto K, Gold PW (2002) Multiple feedback mechanisms activating corticotropin-releasing hormone system in the brain during stress. Pharmacol Biochem Behav 73:147-158.

Mantsch JR, Saphier D, Goeders NE (1998) Corticosterone facilitates the acquisition of cocaine self-administration in rats: opposite effects of the type II glucocorticoid receptor agonist dexamethasone. J Pharmacol Exp Ther 287:72-80.

McEwen BS (2007) Physiology and neurobiology of stress and adaptation: central role of the brain. Physiol Rev 87:873-904.

McEwen BS, Weiss JM, Schwartz LS (1968) Selective retention of corticosterone by limbic structures in rat brain. Nature 220:911-912.

Miller AL, Webb MS, Copik AJ, Wang Y, Johnson BH, Kumar R, Thompson EB (2005) p38 Mitogen-activated protein kinase (MAPK) is a key mediator in glucocorticoid-induced apoptosis of lymphoid cells: correlation between p38 MAPK activation and site-specific phosphorylation of the human glucocorticoid receptor at serine 211. Mol Endocrinol 19:1569-1583.

Nephew BC, Lovelock DF, Bridges RS (2008) The progesterone receptor and parental behavior in juvenile rats. Dev Psychobiol 50:535-541.

Nihalani ND, Schwartz TL (2007) Mifepristone, a glucocorticoid antagonist for the potential treatment of psychotic major depression. Curr Opin Investig Drugs 8:563-569.

Noguchi T, Makino S, Matsumoto R, Nakayama S, Nishiyama M, Terada Y, Hashimoto K (2010) Regulation of glucocorticoid receptor transcription and nuclear translocation during single and repeated immobilization stress. Endocrinology 151:4344-4355.

O'Callaghan MJ, Croft AP, Jacquot C, Little HJ (2005) The hypothalamopituitaryadrenal axis and alcohol preference. Brain Res Bull 68:171-178.

O’Dell LE, Roberts AJ, Smith RT, Koob GF (2004) Enhanced alcohol selfadministration after intermittent versus continuous alcohol vapor exposure. Alcohol Clin Exp Res 28:1676-1682.

Oomen CA, Mayer JL, de Kloet ER, Joëls M, Lucassen PJ (2007) Brief treatment with the glucocorticoid receptor antagonist mifepristone normalizes the reduction in neurogenesis after chronic stress. Eur J Neurosci 26:3395-3401.

Peeters BW, Tonnaer JA, Groen MB, Broekkamp CL, van der Voort HA, Schoonen WG, Smets RJ, Vanderheyden PM, Gebhard R, Ruigt GS (2004) Glucocorticoid receptor antagonists: new tools to investigate disorders characterized by cortisol hypersecretion. Stress 7:233-241.

Piazza PV, Le Moal M (1998) The role of stress in drug self-administration. Trends Pharmacol Sci 19:67-74.

Ponomarev I, Wang S, Zhang L, Harris RA, Mayfield RD (2012) Gene coexpression networks in human brain identify epigenetic modifications in alcohol dependence. J Neurosci 32:1884-1897.

Rasmussen DD, Boldt BM, Bryant CA, Mitton DR, Larsen SA, Wilkinson CW (2000) Chronic daily ethanol and withdrawal: 1. Long-term changes in the hypothalamo-pituitary-adrenal axis. Alcohol Clin Exp Res 24:1836-1849.

Reichardt HM, Schütz G (1998) Glucocorticoid signalling-multiple variations of a common theme. Mol Cell Endocrinol 146:1-6.

Richardson HN, Lee SY, O’Dell LE, Koob GF, Rivier CL (2008) Alcohol self-administration acutely stimulates the hypothalamic-pituitaryadrenal axis, but alcohol dependence leads to a dampened neuroendocrine state. Eur J Neurosci 28:1641-1653.

Ridder S, Chourbaji S, Hellweg R, Urani A, Zacher C, Schmid W, Zink M, Hörtnagl H, Flor H, Henn FA, Schütz G, Gass P (2005) Mice with genetically altered glucocorticoid receptor expression show altered sensitivity for stress-induced depressive reactions. J Neurosci 25:6243-6250.

Rimondini R, Sommer W, Heilig M (2003) A temporal threshold for induction of persistent alcohol preference: behavioral evidence in a rat model of intermittent intoxication. J Stud Alcohol 64:445-449.

Ritter H, Antonova L, Mueller CR (2012) The unliganded glucocorticoid receptor positively regulates the tumour suppressor gene BRCA1 through GABP beta. Mol Cancer Res 10:558-569.

Roberts AJ, Lessov CN, Phillips TJ (1995) Critical role for glucocorticoid receptors in stress- and ethanol-induced locomotor sensitization. J Pharmacol Exp Ther 275:790-797.

Roberts AJ, Heyser CJ, Cole M, Griffin P, Koob GF (2000) Excessive ethanol drinking following a history of dependence: animal model of allostasis. Neuropsychopharmacology 22:581-594.

Samson HH (1986) Initiation of ethanol reinforcement using a sucrosesubstitution procedure in food- and water-sated rats. Alcohol Clin Exp Res 10:436-442.

Sarabdjitsingh RA, Conway-Campbell BL, Leggett JD, Waite EJ, Meijer OC, de Kloet ER, Lightman SL (2010) Stress responsiveness varies over the ultradian glucocorticoid cycle in a brain-region-specific manner. Endocrinology 151:5369-5379. 
Schneider JS, Stone MK, Wynne-Edwards KE, Horton TH, Lydon J, O’Malley B, Levine JE (2003) Progesterone receptors mediate male aggression toward infants. Proc Natl Acad Sci U S A 100:2951-2956.

Schulteis G, Markou A, Cole M, Koob GF (1995) Decreased brain reward produced by ethanol withdrawal. Proc Natl Acad Sci U S A 92:58805884.

Simms JA, Haass-Koffler CL, Bito-Onon J, Li R, Bartlett SE (2012) Mifepristone in the central nucleus of the amygdala reduces yohimbine stressinduced reinstatement of ethanol-seeking. Neuropsychopharmacology 37:906-918.

Sinha R, Fox HC, Hong KI, Hansen J, Tuit K, Kreek MJ (2011) Effects of adrenal sensitivity, stress- and cue-induced craving, and anxiety on subsequent alcohol relapse and treatment outcomes. Arch Gen Psychiatry 68:942-952.

Sommer WH, Rimondini R, Hansson AC, Hipskind PA, Gehlert DR, Barr CS, Heilig MA (2008) Upregulation of voluntary alcohol intake, behavioral sensitivity to stress, and amygdala crhrl expression following a history of dependence. Biol Psychiatry 63:139-145.

Uhart M, Wand GS (2009) Stress, alcohol and drug interaction: an update of human research. Addict Biol 14:43-64.

Valdez GR, Roberts AJ, Chan K, Davis H, Brennan M, Zorrilla EP, Koob GF (2002) Increased ethanol self-administration and anxiety-like behavior during acute ethanol withdrawal and protracted abstinence: regulation by corticotropin-releasing factor. Alcohol Clin Exp Res 26:1494-1501.

Vendruscolo LF, Gueye AB, Darnaudéry M, Ahmed SH, Cador M (2010) Sugar overconsumption during adolescence selectively alters motivation and reward function in adult rats. PLoS One 5:e9296.

Verhoog NJ, Du Toit A, Avenant C, Hapgood JP (2011) Glucocorticoid- independent repression of tumor necrosis factor (TNF) alpha-stimulated interleukin (IL)-6 expression by the glucocorticoid receptor: a potential mechanism for protection against an excessive inflammatory response. J Biol Chem 286:19297-19310.

Walker BM, Koob GF (2007) The gamma-aminobutyric acid-B receptor agonist baclofen attenuates responding for ethanol in ethanol-dependent rats. Alcohol Clin Exp Res 31:11-18.

Wallace AD, Cidlowski JA (2001) Proteasome-mediated glucocorticoid receptor degradation restricts transcriptional signaling by glucocorticoids. J Biol Chem 276:42714-42721.

Wei Q, Lu XY, Liu L, Schafer G, Shieh KR, Burke S, Robinson TE, Watson SJ, Seasholtz AF, Akil H (2004) Glucocorticoid receptor overexpression in forebrain: a mouse model of increased emotional lability. Proc Natl Acad Sci U S A 101:11851-11856.

Wolffgramm J, Heyne A (1995) From controlled drug intake to loss of control: the irreversible development of drug addiction in the rat. Behav Brain Res 70:77-94.

Yang X, Wang S, Rice KC, Munro CA, Wand GS (2008) Restraint stress and ethanol consumption in two mouse strains. Alcohol Clin Exp Res 32:840-852.

Young EA, Abelson J, Lightman SL (2004) Cortisol pulsatility and its role in stress regulation and health. Frontiers in Neuroendocrinology 25:69-76.

Zhao Y, Weiss F, Zorrilla EP (2007) Remission and resurgence of anxietylike behavior across protracted withdrawal stages in ethanol-dependent rats. Alcohol Clin Exp Res 31:1505-1515.

Zorrilla EP, Valdez GR, Weiss F (2001) Changes in levels of regional CRFlike-immunoreactivity and plasma corticosterone during protracted drug withdrawal in dependent rats. Psychopharmacology (Berl) 158:374-381. 